\title{
Work stress and sleep disturbances among internship nursing students
}

\author{
Samah Rabei, Ghada Mourad and Alaa El Din Hamed
}

\begin{abstract}
Background: Nurses are one of the most vulnerable professional groups to occupational stress and sleep problem. A relation between occupational stress and sleep problem is suggested and needs to be investigated. The aim of this descriptive study is designed to assess work stress and sleep disturbances among internship nursing students and the relation between them.

Results: A total of 95 nursing internship students were subjected to Pittsburgh Sleep Quality Index (PSQI) and Expanded Nursing Stress Scale (ENSS). The greater the number of patients a nurse cares for, the greater the nursing stress. Greater number and duration of shifts worsen sleep. Problems related to peers worsen sleep quality. Demanding patients and their families lessen the actual sleep duration of nurses. Demanding patients and their families, work load, discrimination, and uncertainty regarding treatment lessen the habitual sleep efficiency of nurses.
\end{abstract}

Conclusion: Nurses with higher workload experience more sleep disturbances.

\section{Background}

Nursing is considered as a stressful occupation. Stress has an implication for health and the satisfaction level of the nurses involved which eventually has an impact on the quality of care for the patients they attend to. It is financially costly to any health care organization. Negative outcomes of job stress among nurses include absenteeism and staff turnover; also, stress is associated with impaired individual functioning in the workplace, decline in overall quality of care, reduced efficiency, decreased capacity to perform, dampened initiative, reduced interest in working, increased rigidity of thought, a lack of concern for the organization and colleagues, and a loss of responsibility [1]. Description of the nursing internship is the transitional program of new graduated; they last longer and are more comprehensive than traditional hospital orientation programs for new staff nurses. The goals of nursing internship are to ease the transition from the student role to the staff nurse role and to guide new nurses in developing basic and specialized nursing skills [2]. Sleep disturbance in nurses represent highly common phenomena that, in severe forms, can interfere

* Correspondence: samahrabe@yahoo.com

Helwan University, Ain Helwan, Helwan, Cairo, Egypt with daily activities and work performance. It is estimated that more than $25 \%$ of nurses experience significant sleep problem during the day. National sleep disorder research reported that $12 \mathrm{~h}$ shifts are associated with less effective performance, and there is very large, strong body of evidence showing that insufficient sleep has adverse effect on cognition, performance, and mood of registered nurses [3]. In a study analyzing the association between stress and life quality, they reported that there is a correlation between stress and sleep disturbance [4]. In another study developed at seven hospitals in Shanghai, the authors observed that the stress at work was correlated to the impairment of the nurses' sleep $[5,6]$.

\section{Methods}

(A) Setting: in College of Nursing Helwan University, Badr University Hospital, Ain-Shams University Hospital, Wadi El Nil Hospital, El Salam International Hospital, and Nile Badrawy Hospital 
(B) Subject: 95 students in the five hospitals collected in a stratified random method for a descriptive design

(C) Timetable: between June and December 2016

(D) Tools for data collection: demographic data questionnaire, Pittsburgh Sleep Quality Index (PSQI) and Expanded Nursing Stress Scale (ENSS) were used.

- Sociodemographic Questionnaire: developed by the researchers which includes age, sex, religion, marital status, and monthly income.

- The Pittsburgh Sleep Quality Index (PSQI): developed by Buysse et al. [7] and revised by Smyth [8]. It consists of seven domains: sleep quality, latency, duration, efficiency, nigh sleep disturbance, use of sleep medication, and daytime sleepiness over the last month. Each scored from 0 to 3, which sum results in a global score that may range from 0 to 21 . Scores above 5 suggest poor sleep quality, and igher scores indicate sleep of lowest quality.

- Expanded Nursing Stress Scale (ENSS): 36 developed by Gray-Toft and Anderson [9] and revised by French [10]. It contains 57 items in nine subscales: death and dying, conflict with physicians, inadequate emotional preparation, problems relating to peers, problems relating to supervisors, work load, uncertainty concerning treatment, patients and their families, and discrimination. The questionnaire is a 57-item 5point Likert scale. The responses were "never stressful" (1), "occasionally stressful" (2), "Frequently stressful" (4), "extremely stressful" (4), and does not apply (5). The does not apply score of (5) was considered (0) in the statistical analysis. The higher the score, the more the respondent agreed that the situation was stressful. A total and sub-scale mean score is derived from this instrument which ranges from 0 to 4 . There are no specific cut scores or published mean norms for the ENSS that determine whether an individual is stressed or not. However, higher scores indicate higher levels of stress.

(E) Statistical analysis was done using SPSS version 14.

\section{Results (Tables 1, 2, 3, 4, 5, 6, 7, 8, 9 and 10)}

\section{Discussion}

The percentage of nurses in the current study who scored global sum of 8 PSQI more than 5 points (considered to have sleep disturbance) is $90.53 \%$. Most studies showed a lower prevalence of sleep disturbance
Table 1 Distribution of demographic characteristics of the studied nurses

\begin{tabular}{llll}
\hline Demographic & & Number & Percent \\
\hline Sex & Male & 32 & 34 \\
& Female & 63 & 66 \\
Religion & Muslim & 87 & 92 \\
& Christian & 8 & 8 \\
Marital status & Single & 61 & 64 \\
& Engaged & 23 & 24 \\
Residence & Married & 11 & 12 \\
& Urban & 50 & 52 \\
Income & Rural & 45 & 48 \\
& Sufficient & 70 & 74 \\
\hline
\end{tabular}

than this study. Two studies showed only $6.5 \%$ and $27.8 \%$ of subjects self-reporting as getting poor sleep $[11,12]$. However, there are other studies that showed a prevalence of sleep disturbance of more than 50\% $[13,14]$. Our findings are nearly close to the results of a study which reported that $72 \%$ of nurses selfreported as getting insufficient sleep [15]. The extreme difference in the results maybe because of the use of different questionnaires as measures and that the studies of sleep quality were conducted in in different cultures with different conditions of work and

Table 2 Distribution of work conditions for the studied nurses

\begin{tabular}{llll}
\hline Work conditions & & Number & Percent \\
\hline Hospital type & Governmental & 32 & 34 \\
& Private & 63 & 66 \\
Shifts/week & 3 shifts & 27 & 28 \\
& 4 shifts & 41 & 44 \\
Hours/shift & $>4$ shifts & 27 & 28 \\
& 6 h & 2 & 2 \\
Night shifts/week & 12 h & 90 & 95 \\
& 24 h & 3 & 3 \\
Nurse patient ratio & $3-4$ & 54 & 56 \\
& $>4$ & 26 & 27 \\
& 1 for 1 to 2 & 15 & 17 \\
Payment & 1 for $>4$ & 12 & 39 \\
& Acceptable & 64 & 13 \\
& Not & 31 & 67 \\
\hline
\end{tabular}

Nursing stress as to total score of ENSS (7.37\% severe stress, $58.95 \%$ moderate stress, $33.68 \%$ mild stress) 
Table 3 Percent of total expanded nursing stress sub-scales score $(n=95)$

\begin{tabular}{|c|c|c|c|c|c|}
\hline & $\begin{array}{l}\text { Never } \\
\text { stressed } \\
(\%)\end{array}$ & $\begin{array}{l}\text { Occasionally } \\
\text { stressed (\%) }\end{array}$ & $\begin{array}{l}\text { Frequently } \\
\text { stressed } \\
(\%)\end{array}$ & $\begin{array}{l}\text { Extremely } \\
\text { stressed } \\
(\%)\end{array}$ & $\begin{array}{l}\text { Always } \\
\text { occurring } \\
\text { stress (\%) }\end{array}$ \\
\hline $\begin{array}{l}\text { A. Death and } \\
\text { dying }\end{array}$ & 19.6 & 34.3 & 23.75 & 11.6 & 10.75 \\
\hline $\begin{array}{l}\text { B. Conflict } \\
\text { with physician }\end{array}$ & 23.6 & 30.8 & 26 & 10.8 & 8.8 \\
\hline $\begin{array}{l}\text { C. Inadequate } \\
\text { emotional } \\
\text { preparation }\end{array}$ & 22.67 & 40.67 & 23 & 9.33 & 4.33 \\
\hline $\begin{array}{l}\text { D. Problems } \\
\text { related to } \\
\text { supervisor }\end{array}$ & 17.5 & 34.3 & 26.5 & 14.3 & 7.2 \\
\hline $\begin{array}{l}\text { E. Problems } \\
\text { related to } \\
\text { peers }\end{array}$ & 25.1 & 31.9 & 27 & 12 & 4 \\
\hline F. Work load & 18.6 & 32.8 & 26.4 & 13.1 & 9.1 \\
\hline $\begin{array}{l}\text { G. Uncertainty } \\
\text { concerning } \\
\text { treatment }\end{array}$ & 13.2 & 35.34 & 27.5 & 16 & 8 \\
\hline $\begin{array}{l}\text { H. Patients } \\
\text { and their } \\
\text { families }\end{array}$ & 16.6 & 37.3 & 26.7 & 12.1 & 7.3 \\
\hline I. Discrimination & 16.67 & 34.33 & 30.33 & 10 & 8.67 \\
\hline Total & 19.3 & 34.6 & 26.4 & 12.13 & 7.57 \\
\hline
\end{tabular}

resources. It may also be because people suffer from a greater stress level compared with the past years, because of the increasing demand of service. Results of the study shows that demographic circumstances do not have a significant relation neither to sleep quality nor work stress. This is consistent with a study which

Table 4 Percent of sub-scales of Pittsburgh Sleep Quality Index $(n=95)$

\begin{tabular}{|c|c|c|c|c|c|}
\hline $\begin{array}{l}\text { PSQI sub } \\
\text { scales }\end{array}$ & Score & $\begin{array}{l}\text { Not during } \\
\text { the past } \\
\text { month }(0) \\
(\%)\end{array}$ & $\begin{array}{l}\text { Less than } \\
\text { once a } \\
\text { week (1) } \\
(\%)\end{array}$ & $\begin{array}{l}\text { Once or } \\
\text { twice a } \\
\text { week (2) } \\
(\%)\end{array}$ & $\begin{array}{l}\text { Three or } \\
\text { more times } \\
\text { a week (3) } \\
(\%)\end{array}$ \\
\hline $\begin{array}{l}\text { Subjective sleep } \\
\text { quality }\end{array}$ & & 11.6 & 45.3 & 28.4 & 14.7 \\
\hline Sleep latency & & 20 & 33.7 & 29.5 & 16.8 \\
\hline $\begin{array}{l}\text { Actual sleep } \\
\text { duration }\end{array}$ & & 10.5 & 34.7 & 45.3 & 9.5 \\
\hline $\begin{array}{l}\text { Habitual sleep } \\
\text { efficiency }\end{array}$ & & 60 & 31.6 & 6.3 & 2.1 \\
\hline $\begin{array}{l}\text { Sleep } \\
\text { disturbances }\end{array}$ & & 2.1 & 62.1 & 35.8 & 0.00 \\
\hline $\begin{array}{l}\text { Use of sleeping } \\
\text { medication }\end{array}$ & & 70.5 & 16.8 & 9.5 & 3.2 \\
\hline $\begin{array}{l}\text { Daytime } \\
\text { dysfunction }\end{array}$ & & 16.8 & 37.9 & 27.4 & 17.9 \\
\hline Total & & 27.35 & 37.44 & 26.03 & 8.87 \\
\hline
\end{tabular}

Table 5 Relationship between demographic data and level of stress manifestation

\begin{tabular}{|c|c|c|c|c|c|c|c|c|}
\hline \multirow{3}{*}{$\begin{array}{l}\text { Demographic } \\
\text { data }\end{array}$} & \multicolumn{6}{|c|}{ Total level of stress manifestation } & \multirow[t]{3}{*}{$x 2$} & \multirow[t]{3}{*}{$P$ value } \\
\hline & \multicolumn{2}{|c|}{ Mild } & \multicolumn{2}{|c|}{ Moderate } & \multicolumn{2}{|c|}{ Severe } & & \\
\hline & No. & $\%$ & No. & $\%$ & No. & $\%$ & & \\
\hline Sex & & & & & & & .890 & $\geq 0.05$ \\
\hline Male & 9 & 28.12 & 21 & 65.63 & 2 & 6.25 & & \\
\hline Female & 23 & 36.51 & 35 & 55.56 & 5 & 7.93 & & \\
\hline Religion & & & & & & & 1.453 & $\geq 0.05$ \\
\hline Muslim & 28 & 32.2 & 52 & 59.8 & 7 & 8 & & \\
\hline Christian & 4 & 50 & 4 & 50 & 0 & 0 & & \\
\hline Marital status & & & & & & & 9.233 & $\geq 0.05$ \\
\hline Single & 26 & 41.9 & 31 & 50 & 5 & 8.1 & & \\
\hline Engaged & 2 & 9.1 & 19 & 86.4 & 1 & 4.5 & & \\
\hline Married & 4 & 36.4 & 6 & 54.5 & 1 & 9.1 & & \\
\hline Residence & & & & & & & 1.546 & $\geq 0.05$ \\
\hline Urban & 15 & 29.4 & 31 & 60.8 & 5 & 9.8 & & \\
\hline Rural & 17 & 38.64 & 25 & 56.82 & 2 & 4.54 & & \\
\hline Monthly income & & & & & & & 4.082 & $\geq 0.05$ \\
\hline Sufficient & 27 & 38.6 & 37 & 52.9 & 6 & 8.57 & & \\
\hline Insufficient & 5 & 20 & 19 & 76 & 1 & 4 & & \\
\hline
\end{tabular}

Table 6 Relationship between demographic data and sleep quality

\begin{tabular}{|c|c|c|c|c|c|c|}
\hline \multirow{3}{*}{$\begin{array}{l}\text { Demographic } \\
\text { data }\end{array}$} & \multicolumn{4}{|c|}{ Total sleep quality } & \multirow[t]{3}{*}{$x 2$} & \multirow[t]{3}{*}{$P$ value } \\
\hline & \multicolumn{2}{|c|}{ Good } & \multicolumn{2}{|c|}{ Poor } & & \\
\hline & No. & $\%$ & No. & $\%$ & & \\
\hline Sex & & & & & .585 & $\geq 0.05$ \\
\hline Male & 2 & 6.2 & 30 & 93.8 & & \\
\hline Female & 7 & 11.1 & 56 & 88.9 & & \\
\hline Religion & & & & & .093 & $\geq 0.05$ \\
\hline Muslim & 8 & 9.2 & 79 & 90.8 & & \\
\hline Christian & 1 & 12.5 & 7 & 87.5 & & \\
\hline Marital status & & & & & .863 & $\geq 0.05$ \\
\hline Single & 7 & 11.3 & 55 & 88.7 & & \\
\hline Engaged & 1 & 9.5 & 21 & 95.5 & & \\
\hline Married & 1 & 4.1 & 10 & 90.9 & & \\
\hline Residence & & & & & .341 & $\geq 0.05$ \\
\hline Urban & 4 & 7.8 & 47 & 92.2 & & \\
\hline Rural & 5 & 11.4 & 39 & 88.6 & & \\
\hline Monthly income & & & & & 3.551 & $\geq 0.05$ \\
\hline Sufficient & 9 & 12.9 & 61 & 87.1 & & \\
\hline Insufficient & 0 & 0 & 25 & 100 & & \\
\hline
\end{tabular}


Table 7 Relationship between work conditions of studied sample and level of stress

\begin{tabular}{|c|c|c|c|c|c|c|c|c|c|}
\hline \multirow[t]{3}{*}{ Work conditions } & & \multirow[b]{3}{*}{ No. } & \multicolumn{5}{|c|}{ Total level of stress manifestation } & \multirow[t]{3}{*}{$x 2$} & \multirow[t]{3}{*}{$P$ value } \\
\hline & & & \multirow{2}{*}{$\begin{array}{l}\text { Mild } \\
\%\end{array}$} & \multicolumn{2}{|c|}{ Moderate } & \multicolumn{2}{|c|}{ Severe } & & \\
\hline & & & & No. & $\%$ & No. & $\%$ & & \\
\hline \multirow[t]{2}{*}{ Hospital type } & Governmental & 10 & 30.3 & 19 & 57.58 & 4 & 12.12 & 1.738 & $\geq 0.05$ \\
\hline & Private & 22 & 35.48 & 37 & 59.68 & 3 & 4.84 & & \\
\hline \multirow[t]{3}{*}{ Shift number/week } & 3 shifts/week & 10 & 35.7 & 15 & 53.57 & 3 & 10.7 & 1.844 & $\geq 0.05$ \\
\hline & 4 shifts/week & 15 & 37.5 & 23 & 57.5 & 2 & 5 & & \\
\hline & > 4 shifts/week & 7 & 25.93 & 18 & 66.67 & 2 & 7.41 & & \\
\hline \multirow[t]{3}{*}{ Shift hours } & $6 \mathrm{~h} / \mathrm{shift}$ & 1 & 50 & 0 & 0 & 1 & 50 & 6.602 & $\geq 0.05$ \\
\hline & $12 \mathrm{~h} / \mathrm{shift}$ & 30 & 33.33 & 54 & 60 & 6 & 6.67 & & \\
\hline & $24 \mathrm{~h} / \mathrm{shift}$ & 1 & 33.33 & 2 & 66.67 & 0 & & & \\
\hline \multirow[t]{3}{*}{ Night shifts/week } & 1-2/week & 21 & 38.18 & 29 & 52.72 & 5 & 9.1 & 2.266 & $\geq 0.05$ \\
\hline & 3-4/week & 7 & 28 & 17 & 68 & 1 & 4 & & \\
\hline & $>4 /$ week & 4 & 26.67 & 10 & 66.67 & 1 & 6.67 & & \\
\hline \multirow[t]{3}{*}{ Nurse/patient ratio } & Nurse/1-2 & 19 & 51.35 & 13 & 35.14 & 5 & 13.51 & 14.846 & $\leq 0.01 * *$ \\
\hline & Nurse/3-4 & 3 & 23.1 & 10 & 76.92 & 0 & 0 & & \\
\hline & Nurse/> 4 & 10 & 22.22 & 33 & 73.34 & 2 & 4.44 & & \\
\hline \multirow[t]{2}{*}{ Payment } & Acceptable & 22 & 34.92 & 38 & 60.32 & 3 & 4.76 & 1.869 & $\geq 0.05$ \\
\hline & Non acceptable & 10 & 31.25 & 18 & 56.25 & 4 & 12.5 & & \\
\hline
\end{tabular}

**High statistical significance

Table 8 Relationship between work condition of studied sample and level of sleep quality

\begin{tabular}{|c|c|c|c|c|c|c|c|}
\hline & \multirow{3}{*}{$\begin{array}{l}\text { Work } \\
\text { conditions }\end{array}$} & \multirow[b]{3}{*}{ No. } & \multicolumn{3}{|c|}{ Total sleep quality } & \multirow[t]{3}{*}{$x 2$} & \multirow[t]{3}{*}{$P$ value } \\
\hline & & & \multirow{2}{*}{$\begin{array}{l}\text { Good } \\
\%\end{array}$} & \multicolumn{2}{|c|}{ Poor } & & \\
\hline & & & & No. & $\%$ & & \\
\hline \multirow[t]{2}{*}{ Hospital type } & Governmental & 4 & 12.1 & 29 & 87.9 & 0.413 & $\geq 0.05$ \\
\hline & Private & 5 & 8.1 & 57 & 91.9 & & \\
\hline \multirow[t]{3}{*}{ Shift number/week } & 3 shifts/week & 2 & 7.1 & 26 & 92.9 & 6.008 & $\leq 0.05^{*}$ \\
\hline & 4 shifts/week & 7 & 17.5 & 33 & 82.5 & & \\
\hline & $>4$ shifts/week & 0 & 0 & 27 & 100 & & \\
\hline \multirow[t]{3}{*}{ Shift hours } & $6 \mathrm{~h} / \mathrm{shift}$ & 1 & 50 & 2 & 50 & 6.123 & $\leq 0.05^{*}$ \\
\hline & $12 \mathrm{~h} / \mathrm{shift}$ & 7 & 7.8 & 90 & 92.2 & & \\
\hline & $24 \mathrm{~h} / \mathrm{shift}$ & 1 & 33.3 & 3 & 66.7 & & \\
\hline \multirow[t]{3}{*}{ Night shifts/week } & 1-2/week & 7 & 12.7 & 48 & 87.3 & 1.690 & $\geq 0.05$ \\
\hline & 3-4/week & 1 & 4 & 24 & 96 & & \\
\hline & $>4$ shifts/week & 1 & 6.7 & 14 & 93.3 & & \\
\hline \multirow[t]{3}{*}{ Nurse/patient ratio } & Nurse/1-2 & 5 & 13.5 & 32 & 86.5 & 1.166 & $\geq 0.05$ \\
\hline & Nurse/3-4 & 1 & 7.7 & 12 & 92.3 & & \\
\hline & Nurse/> 4 & 3 & 6.7 & 42 & 93.3 & & \\
\hline \multirow[t]{2}{*}{ Shift payment } & Acceptable & 5 & 7.9 & 58 & 92.1 & .515 & $\geq 0.05$ \\
\hline & Not & 4 & 12.5 & 28 & 87.5 & & \\
\hline
\end{tabular}


Table 9 Relationship between level of stress and sleep disturbance

\begin{tabular}{|c|c|c|c|c|c|c|}
\hline \multirow{3}{*}{$\begin{array}{l}\text { Level of } \\
\text { stress }\end{array}$} & \multirow[b]{3}{*}{ No. } & \multicolumn{3}{|c|}{ Total sleep quality } & \multirow[t]{3}{*}{$x 2$} & \multirow[t]{3}{*}{$P$ value } \\
\hline & & \multirow{2}{*}{$\begin{array}{l}\text { Good } \\
\%\end{array}$} & \multicolumn{2}{|c|}{ Poor } & & \\
\hline & & & No. & $\%$ & & \\
\hline Mild & 5 & 15.6 & 27 & 84.4 & 2.707 & $\geq 0.05$ \\
\hline Moderate & 3 & 5.4 & 53 & 94.6 & & \\
\hline Severe & 1 & 14.3 & 6 & 85.7 & & \\
\hline
\end{tabular}

assumed that none of the socio-demographic variables had a statistical impact on sleep quality in nursing students [16].

In this study, one of the findings is that greater number and duration of shifts per week worsens nurses' sleep quality. This parallels the results of a study which assumed that there is a significant relationship between number of hours per shift and stress [17]. As regard to nurse-patient ratio, nearly half of the studied subjects worked as one nurse for more than four patients, while more than one third of them working as one nurse for one to two patients. According to the relationship between nurse patient ratio and level of stress, there was a significant statistical relationship which is consistent with previous study $[18,19]$.

As regards the number of night shifts per week, it was noticed that more than two-thirds of the studied subjects (44\%) were working four shifts per week, while more than one quarter $(28 \%)$ of them working three shifts per week and the same percentage working more than four shifts per week. Concerning the relationship between night shifts per week and sleep quality, results of the present study revealed that globally, the relationship between night shifts per week and sleep quality was not significant, which is in the same line with [20]; however, this finding is not in line with previous studies done by $[21,22]$ and more recent study [23] which reported that there is a significant effect of working in night schedule on sleep disturbance as a whole.

Table 10 Correlation between stress and sleep disturbance, total score, and sub-scales

\begin{tabular}{|c|c|c|c|c|c|c|c|c|c|}
\hline Sleep & Stree & $\begin{array}{l}\text { Subjective } \\
\text { sleep quality }\end{array}$ & $\begin{array}{l}\text { Sleep } \\
\text { latency }\end{array}$ & $\begin{array}{l}\text { Actual sleep } \\
\text { duration }\end{array}$ & $\begin{array}{l}\text { Habitual sleep } \\
\text { efficiency }\end{array}$ & $\begin{array}{l}\text { Sleep } \\
\text { disturbances }\end{array}$ & $\begin{array}{l}\text { Use of sleeping } \\
\text { medications }\end{array}$ & $\begin{array}{l}\text { Day time } \\
\text { dysfunction }\end{array}$ & $\begin{array}{l}\text { Total } \\
\text { PSQI }\end{array}$ \\
\hline \multirow[t]{2}{*}{ Death and dying } & $\mathrm{R}$ & 0.116 & 0.064 & -0.065 & 0.138 & 0.081 & 0.144 & -0.099 & 0.092 \\
\hline & $P$ & 0.263 & 0.535 & 0.533 & 0.184 & 0.435 & 0.165 & 0.341 & 0.373 \\
\hline \multirow[t]{2}{*}{$\begin{array}{l}\text { Conflicts with } \\
\text { physicians }\end{array}$} & $\mathrm{R}$ & -0.051 & -0.116 & -0.15 & 0.076 & 0.003 & -0.037 & 0.057 & $\overline{-}-094$ \\
\hline & $P$ & 0.627 & 0.262 & 0.147 & 0.464 & 0.981 & 0.72 & 0.586 & 0.364 \\
\hline \multirow{2}{*}{$\begin{array}{l}\text { Inadequate emotional } \\
\text { preparation }\end{array}$} & $\mathrm{R}$ & 0.04 & 0.017 & 0.073 & 0.039 & 0.044 & 0.132 & 0.026 & 0.06 \\
\hline & $P$ & 0.7 & 0.873 & 0.483 & 0.71 & 0.67 & 0.202 & 0.799 & 0.564 \\
\hline \multirow{2}{*}{$\begin{array}{l}\text { Problems related to } \\
\text { the supervisor }\end{array}$} & $\mathrm{R}$ & -0.075 & 0.091 & 0.003 & 0.201 & -0.072 & -0.048 & 0.029 & 0.027 \\
\hline & $P$ & 0.475 & 0.386 & 0.975 & 0.052 & 0.492 & 0.649 & 0.778 & 0.797 \\
\hline \multirow{2}{*}{$\begin{array}{l}\text { Problems related to } \\
\text { peers }\end{array}$} & $\mathrm{R}$ & -0.226 & -0.049 & 0.014 & 0.231 & -0.119 & -0.043 & 0.116 & 0.026 \\
\hline & $P$ & 0.028 & 0.64 & 0.89 & 0.024 & 0.253 & 0.681 & 0.263 & 0.805 \\
\hline \multirow[t]{2}{*}{ Workload } & $\mathrm{R}$ & -0.079 & 0.012 & 0.048 & 0.304 & -0.044 & 0.07 & 0.056 & 0.066 \\
\hline & $P$ & 0.45 & 0.909 & 0.649 & 0.003 & 0.672 & 0.504 & 0.59 & 0.528 \\
\hline \multirow{2}{*}{$\begin{array}{l}\text { Uncertainty regarding } \\
\text { treatment }\end{array}$} & $\mathrm{R}$ & -0.08 & 0.088 & -0.03 & 0.253 & -0.04 & -0.071 & 0.113 & 0.052 \\
\hline & $\mathrm{P}$ & 0.44 & 0.398 & 0.74 & 0.013 & 0.7 & 0.496 & 0.275 & 0.617 \\
\hline \multirow{2}{*}{$\begin{array}{l}\text { Patients and their } \\
\text { families }\end{array}$} & $\mathrm{R}$ & 0.016 & -0.035 & 0.226 & 0.313 & -0.109 & -0.1 & -0.024 & 0.078 \\
\hline & $P$ & 0.88 & 0.735 & 0.027 & 0.02 & 0.295 & 0.927 & 0.816 & 0.454 \\
\hline \multirow[t]{2}{*}{ Discrimination } & $\mathrm{R}$ & 0.078 & -0.071 & 0.098 & 0.205 & -0.021 & 0.105 & 0.195 & 0.164 \\
\hline & $P$ & 0.455 & 0.495 & 0.347 & 0.046 & 0.839 & 0.31 & 0.058 & 0.113 \\
\hline \multirow[t]{2}{*}{ Total ENSS } & $\mathrm{R}$ & -0.096 & 0.028 & -0.029 & 0.262 & -0.034 & 0.011 & 0.052 & 0.115 \\
\hline & $P$ & 0.353 & 0.791 & 0.782 & 0.01 & 0.745 & 0.914 & 0.614 & 0.269 \\
\hline
\end{tabular}

Greater number of patients a nurse cares for increases the nursing stress

Greater number and duration of shifts worsen sleep. Demanding patients and their families lessen the actual sleep duration of nurses

Demanding patients and their families, workload, discrimination, and uncertainty regarding treatment lessen is the habitual sleep efficiency of nurses. Problems related to peers worsen sleep quality

Boldface indicates statistical significance 


\section{Conclusion}

Designing programs to detect, prevent, manage, and follow up nurses with nursing stress and sleep disturbances, as well as service training and continuing education, should be provided for all nursing internships during working in the hospital, and self-awareness programs should be applied. Organizing seminars, workshops, conferences, debates, and lectures and opening chat channels is essential to create awareness. Policymakers should be addressed to push toward legalizations and importing resources to handle such phenomena.

\section{Acknowledgements}

Not applicable

\section{Consent for participation}

Written informed consent to participate was taken from students participating.

\section{Authors' approval of manuscript}

They all have read and approved the manuscript.

\section{Authors' contributions}

The authors contributed collaboratively in all the steps of this research. The author(s) read and approved the final manuscript.

\section{Funding}

Researchers' expense

\section{Availability of data and materials}

Available

\section{Competing interest}

There are no competing interests.

Ethics approval and consent to participate

It was taken from Helwan University ethical committee meeting 6 on the 13th of January 2016.

\section{Consent for publication}

Written informed consents to publish was taken from students participating

Received: 8 March 2020 Accepted: 31 March 2020

Published online: 24 June 2020

\section{References}

1. Rita AA, Atindanbila S, Portia MN, Abepuoring P (2013) The causes of stress and job satisfaction among nurses at ridge and pan tang hospitals in Ghana. International Journal of Asian Social Science 3(3):762-771

2. Clair M (2013) New graduate nurses' experiences of transition during orientation into critical care, theses and dissertation submitted in partial fulfillment of the requirements for the De gree of doctor of philosophy in nursing at the University of Wisconsin - Milwaukee, paper 378.

3. Ohida, T. et al, 2009: Epidemiological study of nightmare and sleep paralysis among Japanese adolescents. Sleep and Biological Rhythms, 7(3):201-210. https://doi.org/10.1111/J1479-8425.2009.00404.x.

4. Winwood PC, Lushington K (2006) Disentangling the effects of psychological and physical work demands on sleep, recovery and maladaptive chronic stress outcomes within a large sample of Australian nurses. J Adv Nurs 56(6):679-689

5. Li J, Fu H \& Hu Y, 2008: Work stress and impaired sleep in Chinese nurses. In: Conference abstracts of work, stress, and health - healthy and safe work through research, practice and partnerships; 6-8; Washington, DC, USA. Washington DC: NIOSH, SOHP, APA

6. Rocha MCP and De Martino MMF, 2010: Stress and sleep quality of nurses working different hospital shifts, University of sao Paulo nursing school journal; 44(2):279-285.
7. Buysse D, Reynolds C, Monk T, Berman S, Kupfer D (1989) The pittsburgh sleep quality index. A new instrument. Psych research 28(2):193-213

8. Smyth C (2003) The pittsburgh sleep quality index. Medsurg Nurs 12(4):261-262

9. Gray toft \& Andresen (1981) Stress among hospital nursing staff. Social Science and Medicine 15A:539-647

10. French $S$, Lenton R, Walter $V$, Eyles J (2000) An empirical evaluation of expanded nursing stress index. J Nurs Meas 8(2):161-178

11. Karacan I, Thomby J, Anch M (1986) Prevalence of sleep disturbances in a primarily urban Florida county. Social Sciences Medicine 10:239-244

12. Lack L, Miller W \&Turner D, 1988: A survey on sleeping difficulties in an Australian population. Community Health Studies 12, 200-207.

13. Tawanchai J, Waran T (1997) Sleep disturbance among nurses of Songklanagaring hospital. Journal of Psychiatric Association Thailand 42(3): 123-132

14. Almasi H, Saberi HM, Motallebi HM (2006) Prevalence of sleep disorder in nurses working on rotating shifts. J Appl Sci 6(3):644-646

15. Shan M (2008) Factors associated with perceived sleep quality of nurses working on rotating shifts. J Clin Nurs 18:285-293

16. Silva M, Chaves C, Duarte J, Amaral O \& Ferreira M, 2016: Sleep quality determinants among nursing students, Procedia - Social and Behavioral Sciences 217, PP 999 - 1007

17. Hamaideh SH, Ammouri A (2011) Comparing Jordanian nurses' job stressors in stressful and non -stressful clinical areas. Contemp Nurse 37(2):173-187

18. Henry K J, 2014: Nurses' perceptions of nurse staffing and the impact on patient falls, Master of Science in Nursing. Pp 45-65.

19. Namba H, Koike A \& Wakabayashi T, 2012: Effects of a 7:1 patient-to-nurse staffing ratio on nurses' stressors, accumulated fatigue, and intention to continue working, the Japan Society of NursingResearch(4)35.

20. Angelone AM, Mattei A, Sbarbati M, Dlorio F (2011) Prevalence and correlates for self -reported sleep problems among nursing students. Journal of Preventive Medicine and Hygiene 52:201-208

21. Akerstedt $T$, Torsvall $L$ (1981) Shift-dependent well-being and individual differences. Ergonomics 24:265-273

22. Bryden G, Holdstock T (1973) Effects of night duty on sleep patterns of nurses. Psychophysiology 10:36-42

23. Flo E, Pallesen S, Magerøy N, Moen BE, Grønli J, Nordhus I, Bjorvatn B (2012) Shift work disorder in nurses -assessment, prevalence and related health problems. PLoS-One 7(4):e33981

\section{Publisher's Note}

Springer Nature remains neutral with regard to jurisdictional claims in published maps and institutional affiliations.

\section{Submit your manuscript to a SpringerOpen ${ }^{\circ}$ journal and benefit from:}

- Convenient online submission

- Rigorous peer review

- Open access: articles freely available online

High visibility within the field

- Retaining the copyright to your article

Submit your next manuscript at $>$ springeropen.com 